\title{
BILIARY TRACT
}

\section{GPC1 genetic risk further links Hedgehog signalling with pathogenesis of biliary atresia}

"Biliary atresia is the most common identifiable cause of neonatal cholestasis and is the leading cause of liver transplantation in children," states Randolph Matthews, corresponding author of a recent study investigating the pathogenesis of this disease.

"Although advances have been made in our understanding of the mechanisms underlying the fibroinflammatory damage to the biliary tree that occurs in patients with this disorder, uncovering the cause of biliary atresia continues to be a major challenge," he explains.

Biliary atresia might be caused by exposure of a genetically susceptible individual to environmental factors during early infancy. Variants of genes that have a role in hepatobiliary development and/or immunological tolerance are likely candidates for mediating susceptibility. A previous genome-wide association study found a potential region of interest at $2 \mathrm{q} 37$; the investigators in the current study aimed to discover if this region contained any specific biliary atresia susceptibility genes. Analysis of copy number variant increases in patients with biliary atresia $(n=61)$ compared with controls $(n=5,088)$ revealed a marked difference in region $2 \mathrm{q} 37.3$, which corresponded with loss of the gene GPC1. This gene encodes glypican 1 , a heparan sulphate proteoglycan, which has a key role in regulating Hedgehog signalling.

The investigators used zebrafish to determine the mechanisms caused by deletion of GPC1 that lead to biliary atresia. Knockdown of $g p c 1$ in zebrafish led to developmental biliary defects. Exposure of these $g p c 1$ morphants to a Hedgehog antagonist partially rescued the phenotype and injection of recombinant Hedgehog into wild-type zebrafish led to similar biliary defects as observed in the morphants, providing evidence that Hedgehog signalling is important in this disorder. The researchers then went back to patients and found lower levels of apical GPC1 in cholangiocytes in patients with biliary atresia than in controls.

"The most significant findings were that we identified a specific gene associated with biliary atresia, and then using animal models were able to show that disruption of the gene led to biliary defects and overactivation of Hedgehog signalling, as has been shown to occur in patients with this disease," says Matthews. "The ability to test infants for susceptibility to biliary atresia in clinical practice is clearly far off. At this point, it seems that genetic association studies coupled with functional studies might be more helpful in identifying molecular pathways important in biliary atresia pathogenesis."

Katherine Smith

Original article Cui, S. et al. Evidence from human and zebrafish that GPC1 is a biliary atresia susceptibility gene. Gastroenterology doi:10.1053/j.gastro.2013.01.022 